\title{
DOES POLICY UNCERTAINTY AFFECT EQUITY, COMMODITY, INTEREST RATES, AND CURRENCY MARKETS? EVIDENCE FROM CBOE'S VOLATILITY INDEX
}

\author{
Imlak SHAIKH $\mathbb{B D}^{*}$ \\ Department of Accounting and Finance, Management Development Institute Gurgaon, \\ Gurugram, Haryana, India
}

Received 28 August 2019; accepted 23 March 2020

\begin{abstract}
Economic policy drives investment, production, employment, and other macroeconomic indicators of the economy. The study examines the equity, commodity, interest rates, and currency markets, taking into consideration the US economic policy uncertainty (EPU) index. The present work determines the association among policy uncertainty and volatility index, expressed in terms of generalized autoregressive conditional heteroscedasticity and period of empirical work spanning from 2000 to 2018. The results suggest that equity markets' volatility tends to be very high based on a high degree of policy uncertainty. The findings on the commodity market indicate that crude oil and gold prices remain more volatile during the presidential election and financial crisis. One of the essential results shows that the 2000s boom, early credit crunch, Lehman's collapse and recession, and fiscal policy battles have significantly affected the equity, currency, and commodity markets. The interest rates and currency markets have responded considerably to Feds' and EPU index. The empirical outcome provides evidence that implied volatility index is a forward looking expectation of future stock market volatility, and it uncovers that policy uncertainty affects investor sentiment. The present work holds some practical implications for the government to formulate policies to regulate the US market.
\end{abstract}

Keywords: policy uncertainty, equity, commodity, interest rate, foreign exchange, VIX.

JEL Classification: G12, G14, G18.

\section{Introduction}

The Chicago Board Options Exchange's (CBOE) has recently completed 25 years as an exchange for trading into options. VIX is a registered trademark of CBOE and one of the best innovations of the modern financial measure. It is now considered globally as the gauge of the investors' fear. In the last 25 years, VIX and allied products have grown and become the worlds' most acceptable measure of future stock market volatility. Implied volatility ex-

*Corresponding author. E-mails: imlak786@gmail.com; imlak.shaikh@mdi.ac.in 
plains the future stock market volatility for the residual life of the option. According to the "efficient market hypothesis", observed option prices do contain all important market-wide information, and options' implied volatility is an indicator of market efficiency (Christensen \& Prabhala, 1998). Implied volatility is the future stock market volatility and measures the investors' sentiment on a real-time basis (e.g., Whaley, 2000). Higher the uncertainty in the market, more VIX tends to rise. This study supplicates the opportunity to explain the behaviour of options' implied volatility of different 14 markets of the US concerning "economic policy uncertainty" (EPU). Baker et al. (2016) developed a new index of EPU based on newspaper archives - that is, the economic and financial keywords such as "economic/ economy", "uncertain/uncertainty", "congress", "deficit", "federal reserve/Federal Open Market Committee [FOMC]", "gross domestic product", "legislation/regulation" and "Whitehouse", etc. that frequently appear in the newspaper and online portals. It is general wisdom that Wall Street investors closely follow their favourite financial/economic dailies to form the best investment proposal. Baker et al. (2016) built two policy uncertainty indices daily for the US market. The EPU and EMPU indices are daily indices of the US economy and the US financial markets, respectively ${ }^{1}$.

Baker et al. (2016) first employed VIX as the explanatory variable and found bigger stock market volatility and reductions in investment and employment with sensitive policy uncertainty. VIX and EPU indices observed to move together (correlation, 0.58) frequently. The present work is the motivation to extend the effects of policy uncertainty on the VIX index across various asset classes. The study examines 14 implied volatility indices for the US equity, commodity, exchange rate/currency, and treasury markets. The FOMC (2009) and IMF (2012) observed that global financial crises 2008-2009 and fiscal cliff 2012-2013 were caused due to uncertainty about the US and Eurozone monetary policy, and the budgetary and regulatory policies.

Baker et al. (2016) and Christou et al. (2017) studied the effects of EPU at the firm level and stocks in the PacificRim countries. However, our study exclusively focuses on the impact of economic policy uncertainty on implied volatility index (VIX), across various asset classes for the US market. Thus, investigating the effects of scheduled FOMC meeting days, gross domestic product (GDP), and other macroeconomic indicators. This study contributes to investigations into the link between EPU and implied volatility indices. The policy uncertainty index rises significantly during the leading political and economic events and causes the investment and saving behaviour of the economy. EPU and VIX are the measures of the degree of uncertainty, a rise in the EPU index increase the general level of VIX. The EPU-EMPU index and the implied volatility index appear encouraging and substantial transversely in all markets. Our approach to explaining the future stock market volatility is novel in several aspects. First, this is the pioneering attempt to analyse the investors' sentiment, followed by the policy uncertainty index in the US markets. Second, work is very comprehensive using extended time series framework, along with 14 volatility indices dealing with stock, commodity, foreign-exchange (FX), and treasury markets. Third, we consider major macroeconomic indicators such as Federal Open Market Committee meeting day, Gross Domestic

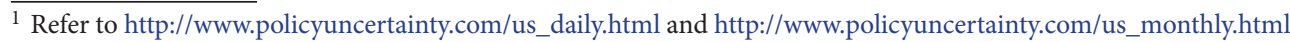


Product report day, and other macroeconomic reports for the US economy. Fourth, the work involved modelling the stock market volatility in the conditional volatility framework and encompassing major historical economic and political events.

The article has been ordered as follows. The section "Introduction" introduces the work and motivation. Section 1 presents the theoretical framework and earlier studies. Section 2 describes data sources and descriptions. Section 3 explains the empirical model building, and Section 4 provides results and discussions. Section, "Conclusions," lists the conclusion and practical implications of the study.

\section{Theoretical framework and literature review}

Pástor and Veronesi $(2012,2013)$ elucidate the relationship between uncertainty and equity market behaviour. Likewise, Baker et al. (2016) build the economic policy uncertainty index, which deals with the policy uncertainty and effects on macroeconomic indicators and equity market volatility, etc. Pástor and Veronesi (2013) develop a general-equilibrium-model and explain that governments' policy uncertainty directs equity risk-premium. The weak economic conditions contribute to a high degree of volatility and correlation. Three types of shocks are responsible for asset price changes: capital, impact, and political. Capital and impact shocks are also identified as a fundamental economic shock. A political shock occurs under the uncertainty of a forthcoming strategy of the government and is orthogonal to shocks of the economy. Political deliberations and discussions of the next regime determine the mandate for extra equity risk-premium (e.g., Witkowska et al., 2019). Risk-premium is measured as "political risk-premiums" linked with representatives' trust around the new administration policy. When economic conditions are stronger political risk-premium remains lower.

The present scholarly work analyses the effects of policy uncertainty on the investors' sentiment gauged in 14 different markets. The market linked risk-premium and policy related uncertainty are powerfully connected. The unfortunate economic circumstances affect not only the market risk-premium but also the returns and volatility of the assets. The volatility in relation to various asset classes will be higher when economic policies are tight and apparent as more diverse. The empirical results support the notion of strict monetary policy, and stringent economic decisions cause a high level of VIX and increased realized volatility.

Ghirelli et al. (2019) develop the economic policy uncertainty of Spain by replicating the mechanism of Baker et al. (2016) and find that the economic policy uncertainty of Baker et al. contributes expected negative sign to the EPU of Spain. Moreover, Degiannakis and Filis (2019) further explore the forecasting of European economic policy uncertainty in relation to global EPU, implied volatility index (VIX), and realized volatility and show that GEPU and VIX hold the highest amount of predictive power to forecast the European EPU. Recently, Huang and Luk (2020) unlike EPU of Spain they construct daily and monthly scale uncertainty index for China, and report that Chines EPU impact equity price negatively and adverse effects on the production and employment. Besides, there has been some recent evidence of the impact of EPU on the US equity market and Bitcoin returns (e.g., Shaikh, 2019a, 2019b, 2020; Wang et al., 2019). 
Ashraf and Shen (2019) also employ the EPU index across major 17 nations to study interest rate and bank lending and find that EPU does have a positive impact on the banks' loan pricing. Unlike economic policy uncertainty index Husted et al. (2019) construct monetary policy uncertainty index (MPU) based on the Federal Reserve policy meetings and actions, they find that MPU causes a decline in the firms' investment and also affects the output and credit creation.

Badshah et al. (2019) studies the effects of economic policy uncertainty on the stock and commodity correlations and hedging strategies, and report that impact is more pronounced under weak economic conditions. Additionally, Alola and Uzune (2020) examine the effects of global EPU on the housing market and agriculture land for the major 15 countries in panel data sets, importantly they show that global EPU affects positively on the housing market whereas land was unresponsive.

Earlier scholarly attempts (e.g., Bali et al., 2017; Chen \& Clement, 2007; Graham et al., 2003; Gábor \& Georgarakos, 2018; Nikkinen \& Sahlström, 2004a, 2004b; Nikkinen et al., 2006; Onan et al., 2014; Husted et al., 2019) document the stock market volatility in the association of macroeconomic indicators and FOMC meetings. They present the movement in the VIX following the macro data and monetary policy uncertainty (MPU). Authors find that due to economic and political uncertainty, the implied volatility index tends to increase prior to the information releases and return normal on the day of press releases. Moreover, studies (e.g., Farka \& Fleissig, 2012; Reinhart \& Simin, 1997; Rigobon \& Sack, 2004; Wang $\&$ Mayes, 2012) examine the effects of uncertainty of the federal policy in terms of minutes of FOMC meetings and show that Feds' policy-rate-change related uncertainty causes significantly to the financial assets.

\section{Data description and descriptive statistics}

The present work examines the 14 volatility indices for the US market under the policy uncertainty of the US economy. The US market volatility indices include equity market, commodity, FX, and interest rates. The sample period ranges from January 2000 to March 2018, depending upon the data availability of all VIX-based volatility indices. We obtain VIX values on a daily scale from the Chicago board of options exchange. The policy uncertainty website provides the values for EPU and EMPU index, which is calculated and disseminated by Baker et al. (2016). Appendix (Table A.1) provides detailed information about various volatility indices and their sample period. Appendix (Table A.2) furnishes data about scheduled announcements of macroeconomic indicators of the US economy. Moreover, the reports of earnings of wages and salary, business employment dynamics, and employment cost index (e.g., Knapkova et al., 2019) (see Table 1).

Table 2 exhibits the coefficient of correlation on various measures of VIX along with the policy uncertainty index. We can see that the correlation between VIX and policy uncertainty index seems to be encouraging and statistically significant. For the entire sample, the correlation coefficient appears to be in a range of $0.20-0.50$. For the calendar years, correlation calculated about 0.70 and statistically significant, and this outcome is in line with the findings of Baker et al. (2016). The correlation coefficient between VIX-EPU and VIX-EMPU calculated respectively, 0.45 and 0.43 positive and statistically significant at $1 \%$ level. In the 
case of crude oil OVX and correlation with the policy uncertainty index, the correlation coefficient appears 0.23 and 0.26 . Likewise, for gold price GVZ, it seems to be 0.32 and 0.24 and statistically significant. The corn and soybean (CIV-SIV) market also exhibits a positive association. Also, the exchange rate volatility of Euro, JPY, and BP shows a significant positive association. The government securities market (TYVIX) also reports a substantial positive correlation followed by policy uncertainty. Figure 1 also shows the association, as mentioned above, between expected stock market volatility and policy uncertainty.

Table 3 summarizes the tests of stationarity of volatility indices and policy uncertainty index. We present a summary of various unit root tests via Levin, Lin, and Chut -stat ; Im, Pesaran and Shin W-stat; ADF-Fisherchi-square; PP-Fisherchi-square. It is apparent from the table that the raw values of VIX, EPU, and EMPU do not exhibit the trend and remain stationary in level.

\section{Empirical model building}

The study underwrites to explore the link between "policy uncertainty" and "markets" expected volatility (VIX). Baker et al. (2016) contribute policy-uncertainty-related indices and uncover the relationship between uncertainty and macroeconomy performance. Moreover, Pástor and Veronesi $(2012,2013)$ explain how uncertainty commands equity risk-premium by developing a general-equilibrium model. Unlike the previous studies (e.g., Baker et al., 2016; Demir et al., 2018; Duan et al., 2018; Hu et al., 2018; Raza et al., 2018), the present work attempts to explain changes in the volatility of the volatility indexes specific to "policy uncertainty". FOMC meetings, GDP report, and other macroeconomic indicators have been considered to show the effects of policy uncertainty on the various asset class. Moreover, policy-uncertainty-related measures, likewise EPU and EMPU, also have been analysed. Uncertainty about the presidential election also causes investor sentiment (Shaikh, 2017), and major economic and political trials also affect market behaviour (Baker et al., 2016). Hence, a set of presidential election years and major historical events are introduced in the empirical model to measure investors' behaviour.

Let $\sigma_{t}^{V I X}$ be the contemporaneous changes in the volatility index and $D_{i t}$ be the dummy variable denoting scheduled macroeconomic announcements. Assume $D_{i t}$ to be 1 on the day of the scheduled macroeconomic announcement, otherwise 0 . The mean equation concerning the effects of uncertainty about monetary policy, production, and other macroeconomic indicators is expressed as follows:

$$
\sigma_{t}^{V I X}=\beta_{0}+\beta_{i} D_{i t}+e_{t},
$$

where $\beta_{0}$ is a conventional intercept term, while $\beta_{i}$ is the slope for the $i^{\text {th }}$-dummies ; $i=F O M C, G D P$, and other macroeconomic indicators; $e_{t}$ is the classical white noise process.

There is an asymmetric relation between returns and volatility. It is established that the underlying asset's index and corresponding VIX are negatively associated. Therefore, in each regression model, an asymmetric return variable on the underlying index has been added as a regressor to control such a relation. Furthermore, in each regression, an autoregressive term $A R(p)$ has been included to control for the autocorrelation. 
The measurement of the volatility of the implied volatility index, it has been expressed in terms of heteroskedastic conditional volatility framework. The eventual purpose of the volatility analysis is to find out the sources of volatility persistence. Engle (2001) rightly pointed out that volatility forecasting using the ARCH/GARCH framework also can be extended when a predetermined or exogenous variable exists. Such as Nana et al. (2013) attempt to derive GARCHX models with exogenous variables. In the GARCHX model, exogenous variables assumed to be functions of stationarity and are the exogenous process. The present study implements $\operatorname{GARCHX}(1,1)$ process as follows:

$$
\begin{gathered}
\sigma_{t}^{V I X}=h_{t} e_{t} ; \\
h_{t}=\omega_{0}+\alpha e_{t-1}^{2}+\beta h_{t-1} .
\end{gathered}
$$

Here, Eq. (3) is the conventional $\operatorname{GARCH}(1,1)$ model, and $\operatorname{GARCHX}(1,1)$ model with exogenous variables has defined as,

$$
h_{t}^{\delta}=g\left(e_{t-1}\right)+u\left(x_{t-1}\right)+c\left(e_{t-1}\right) h_{t-1}^{\delta},
$$

where $h_{t}^{\delta}$ is the conditional variance at time $t,\left\{x_{t-1}\right\}$ represents the exogenous variables such as EPU, EMPU, and other key historical events. $g, c$ and $u$ are the non-negative continuous functions, and $\delta$ indicates a non-negative real number. Alternatively, the GARCHX model in terms of constant $w$ and $\lambda$ coefficient of an exogenous variable is expressed as:

$$
\begin{gathered}
\sigma_{t}^{V I X}=h_{t}(\theta) e_{t} ; \\
h_{t}^{\delta}(\theta)=w+g_{1}\left(e_{t-1} ; \theta^{(1)}\right)+\lambda u_{1}\left(x_{t-1}\right)+c\left(e_{t-1} ; \theta^{(2)}\right) h_{t-1}^{\delta}(\theta) .
\end{gathered}
$$

Hence, $e_{t} \sim N(0,1)$ for the creation of the likelihood function. In GARCHX estimation, the underlying distribution is Gaussian, hence $Z$-statistic is reported with a given standard error. Adjusted $R$-squared is the result of the nested regression of Eq. (1). Table 1 presents variable description and hypothesis, along with expected sign of the regressors.

Table 1. The hypothesis of the model (source: authors' calculation)

\begin{tabular}{|l|c|l|}
\hline \multicolumn{1}{|c|}{ Regressors } & Expected sign & \multicolumn{1}{c|}{ Description/Hypothesis } \\
\hline Intercept & + ve & $\begin{array}{l}\text { The intercept term measures the co-movement in the implied } \\
\text { volatility throughout the non-announcement period. Since, } \\
\text { due to lack of information about the future states of nature, } \\
\text { investors' level of anxiety increases; hence during this period, } \\
\text { volatility rises till the announcement is available (e.g., Nikkinen } \\
\text { \& Sahlström, 2004a, 2004b; Nikkinen et al., 2006). }\end{array}$ \\
\hline FOMC & - ve & $\begin{array}{l}\text { Once the FOMC committee announces the monetary policy } \\
\text { volatility index goes its normal level (e.g., Nikkinen \& } \\
\text { Sahlström, 2004a, 2004b). }\end{array}$ \\
\hline GDP & - ve & $\begin{array}{l}\text { Investors also regard the GDP data for their portfolio planning; } \\
\text { hence on the announcement of GDP report implied volatility } \\
\text { should fall till its normal level. }\end{array}$ \\
\hline
\end{tabular}


End of Table 1

\begin{tabular}{|c|c|c|}
\hline Regressors & Expected sign & Description/Hypothesis \\
\hline $\begin{array}{l}\text { Other } \\
\text { Macroeconomic } \\
\text { Indicators }\end{array}$ & - ve & $\begin{array}{l}\text { For example, CPI/WPI/PPI/IIP and other macroeconomic } \\
\text { indicators on those report days level of implied volatility fall. } \\
\text { The plausible reason is resolving the ambiguity about macro } \\
\text { data. }\end{array}$ \\
\hline $\begin{array}{l}\text { EPU-Economic } \\
\text { Policy Uncertainty }\end{array}$ & $+\mathrm{ve}$ & \multirow{2}{*}{$\begin{array}{l}\text { Uncertainty index and implied volatility index are positively } \\
\text { associated; hence EPU and EMPU should appear positive and } \\
\text { statically significant (e.g., Baker et al., 2016). }\end{array}$} \\
\hline $\begin{array}{l}\text { EMPU-Equity } \\
\text { Market policy } \\
\text { uncertainty }\end{array}$ & + ve & \\
\hline \multicolumn{3}{|c|}{ Major historical Economic and Political events } \\
\hline 2000s boom & + ve & \multirow{4}{*}{$\begin{array}{l}\text { Dummies on those major historical events should appear } \\
\text { positive and statistically significant. These are the major event } \\
\text { during which EPU and volatility index spikes (e.g., Baker et al., } \\
\text { 2016). }\end{array}$} \\
\hline $\begin{array}{l}\text { Early credit } \\
\text { crunch }\end{array}$ & + ve & \\
\hline $\begin{array}{l}\text { Lehman collapse } \\
\text { \& recession }\end{array}$ & + ve & \\
\hline $\begin{array}{l}\text { Fiscal policy } \\
\text { battles }\end{array}$ & + ve & \\
\hline \multicolumn{3}{|c|}{ Presidential Election Year } \\
\hline P.E. Year 2000Q4 & + ve & \multirow{5}{*}{$\begin{array}{l}\text { Dummies on the presidential election should also appear } \\
\text { positive and statistically significant. The fourth quarter of the } \\
\text { respective presidential election causes equity market portfolio } \\
\text { planning. }\end{array}$} \\
\hline P.E. Year 2004Q4 & $+\mathrm{ve}$ & \\
\hline P.E. Year 2008Q4 & $+\mathrm{ve}$ & \\
\hline P.E. Year $2012 \mathrm{Q} 4$ & $+\mathrm{ve}$ & \\
\hline P.E. Year 2016Q4 & + ve & \\
\hline
\end{tabular}

Table 2. Correlation coefficients

\begin{tabular}{|l|c|c|c|c|c|c|c|}
\hline Vol. Index & VIX & VXN & VXO & VXD & VVIX & OIV & OVX \\
\hline EPU & $0.4487 \dagger$ & $0.3529 \dagger$ & $0.4287 \dagger$ & $0.4359 \dagger$ & $0.0849 \dagger$ & 0.0039 & $0.2285 \dagger$ \\
\hline p-value & 0.000 & 0.000 & 0.000 & 0.000 & 0.000 & 0.864 & 0.000 \\
\hline EMPU & $0.4302 \dagger$ & $0.4999 \dagger$ & $0.4593 \dagger$ & $0.4443 \dagger$ & $0.2189 \dagger$ & $0.1421 \dagger$ & $0.2604 \dagger$ \\
\hline p-value & 0.000 & 0.000 & 0.000 & 0.000 & 0.000 & 0.000 & 0.000 \\
\hline Vol. Index & GVZ & SIV & CIV & EUVIX & JYVIX & BPVIX & TYVIX \\
\hline EPU & $0.3201 \dagger$ & $0.2158 \dagger$ & $0.2677 \dagger$ & $0.4347 \dagger$ & $0.3374 \dagger$ & $0.3333 \dagger$ & $0.3386 \dagger$ \\
\hline p-value & 0.000 & 0.000 & 0.000 & 0.000 & 0.000 & 0.000 & 0.000 \\
\hline EMPU & $0.2379 \dagger$ & $0.1496 \dagger$ & $0.1806 \dagger$ & $0.2912 \dagger$ & $0.3240 \dagger$ & $0.2577 \dagger$ & $0.4085 \dagger$ \\
\hline p-value & 0.0000 & 0.0000 & 0.0000 & 0.0000 & 0.0000 & 0.0000 & 0.0000 \\
\hline
\end{tabular}

Note: Table shows the correlation coefficient between various implied volatility indices and EPU and EMPU. $\uparrow$ Significant at $1 \%$ level of significance. 


\begin{tabular}{|c|c|c|c|c|c|c|c|c|c|c|c|}
\hline \multirow{2}{*}{ 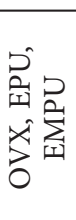 } & \multirow{2}{*}{ 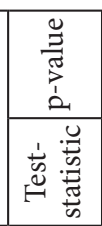 } & \multirow{2}{*}{\begin{tabular}{l}
8 \\
8 \\
0 \\
\multirow{0}{0}{} \\
$\stackrel{\leftrightarrow}{1}$ \\
1
\end{tabular}} & \multirow{2}{*}{$\begin{array}{l}\stackrel{8}{8} \\
\stackrel{0}{0} \\
\vec{\infty} \\
\stackrel{\infty}{1}\end{array}$} & \multirow{2}{*}{ 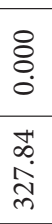 } & \multirow{2}{*}{ 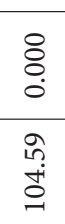 } & \multirow{2}{*}{ 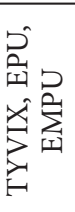 } & \multirow{2}{*}{ 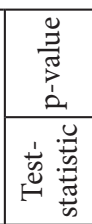 } & \multirow{2}{*}{ 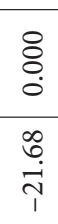 } & \multirow{2}{*}{$\begin{array}{l}8 \\
8 \\
0 \\
0 \\
\infty \\
0 \\
1 \\
1\end{array}$} & \multirow{2}{*}{$\begin{array}{l}8 \\
8 \\
0 \\
10 \\
0 \\
+ \\
0 \\
0\end{array}$} & \multirow{2}{*}{$\begin{array}{l}8 \\
8 \\
0 \\
0 \\
\text { in } \\
\text { in }\end{array}$} \\
\hline & & & & & & & & & & & \\
\hline \multirow{2}{*}{ 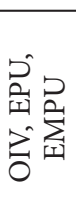 } & 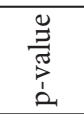 & $\begin{array}{l}8 \\
\vdots \\
0\end{array}$ & \&̊̀. & \&̊․ & $\begin{array}{l}8 \\
8 \\
0\end{array}$ & \multirow{2}{*}{ 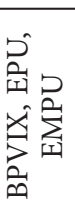 } &  & \&̊̀ & $\begin{array}{l}8 \\
0 \\
0\end{array}$ & $\begin{array}{l}8 \\
0 \\
0\end{array}$ & $\begin{array}{l}8 \\
8 \\
0\end{array}$ \\
\hline & 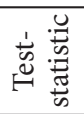 & $\stackrel{m}{\stackrel{m}{T}}$ & 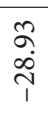 & 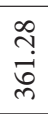 & $\begin{array}{l}\text { nn } \\
\text { ?. } \\
\stackrel{0}{0}\end{array}$ & & 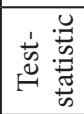 & 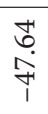 & $\stackrel{\stackrel{2}{n}}{\underset{+}{+}}$ & $\begin{array}{l}20 \\
0 \\
\infty \\
\infty\end{array}$ & $\begin{array}{l}\stackrel{n}{?} \\
\stackrel{\infty}{\infty}\end{array}$ \\
\hline \multirow{2}{*}{  } & 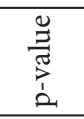 & $\begin{array}{l}8 \\
\circ \\
0\end{array}$ & \&̊े & \&े. & $\begin{array}{l}8 \\
0 \\
0\end{array}$ & \multirow{2}{*}{ 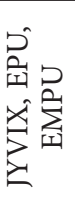 } & 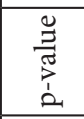 & \&̊े & \&̊․ & $\begin{array}{l}8 \\
8 \\
0\end{array}$ & $\begin{array}{l}8 \\
0 \\
0\end{array}$ \\
\hline &  & $\begin{array}{l}\infty \\
\infty \\
\stackrel{0}{0} \\
i\end{array}$ & $\begin{array}{l}\stackrel{2}{\widehat{~}} \\
\hat{1}\end{array}$ & $\begin{array}{l}\hat{O} \\
\stackrel{\dot{D}}{\infty} \\
\infty\end{array}$ & $\begin{array}{l}\infty \\
0 \\
\infty \\
\infty\end{array}$ & & 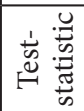 & $\begin{array}{l}0 \\
10 \\
\infty \\
1\end{array}$ & 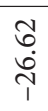 & 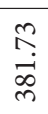 & $\begin{array}{l}\infty \\
\dot{0} \\
= \\
=\end{array}$ \\
\hline \multirow{2}{*}{ 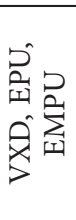 } & 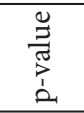 & $\begin{array}{l}8 \\
\circ \\
\circ\end{array}$ & \&̊̀. & ஓे & $\begin{array}{l}8 \\
\stackrel{0}{0} \\
0\end{array}$ & \multirow{2}{*}{ 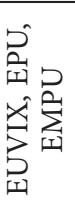 } & 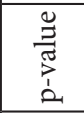 & \&̊․ & $\begin{array}{l}8 \\
8 \\
0\end{array}$ & $\begin{array}{l}8 \\
0 \\
0\end{array}$ & $\begin{array}{l}8 \\
8 \\
0\end{array}$ \\
\hline & 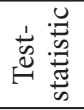 & $\begin{array}{l}\stackrel{\sim}{2} \\
\stackrel{\sim}{1} \\
i\end{array}$ & 异 & $\begin{array}{l}R \\
\infty \\
\infty \\
\infty \\
-1\end{array}$ & $\begin{array}{l}\stackrel{2}{1} \\
\stackrel{n}{n}\end{array}$ & & 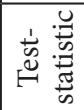 & $\begin{array}{l}\infty \\
\stackrel{0}{0} \\
\infty \\
1\end{array}$ & $\begin{array}{l}\vec{\sigma} \\
\dot{0} \\
i \\
i\end{array}$ & $\begin{array}{l}\infty \\
\infty \\
\dot{0} \\
\infty \\
\infty\end{array}$ & $\begin{array}{l}\text { m} \\
\text { Jू }\end{array}$ \\
\hline \multirow{2}{*}{ 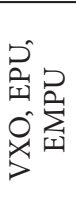 } & 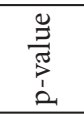 & ஓ̊ & \& & \& & $\begin{array}{l}8 \\
0 \\
0\end{array}$ & \multirow{2}{*}{ 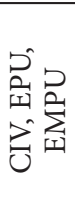 } & 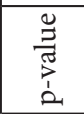 & \& & \& & \& & $\begin{array}{l}8 \\
8 \\
0\end{array}$ \\
\hline & 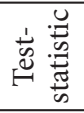 &  & $\begin{array}{l}\stackrel{+}{\infty} \\
\stackrel{1}{\top}\end{array}$ & 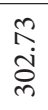 & $\begin{array}{l}\stackrel{0}{1} \\
\stackrel{1}{n}\end{array}$ & & 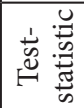 & $\begin{array}{l}\stackrel{8}{\circ} \\
\text { 市 }\end{array}$ &  & $\begin{array}{c}m \\
\infty \\
\infty\end{array}$ & ָे \\
\hline \multirow{2}{*}{ 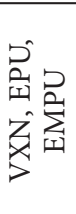 } &  & \begin{tabular}{l}
8 \\
$\circ$ \\
\hdashline
\end{tabular} & $\begin{array}{l}8 \\
8 \\
0\end{array}$ & $\begin{array}{l}8 \\
\vdots \\
0\end{array}$ & $\begin{array}{l}8 \\
8 \\
0\end{array}$ & \multirow{2}{*}{ 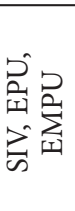 } & 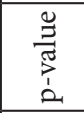 & $\begin{array}{l}8 \\
\vdots \\
0\end{array}$ & $\begin{array}{l}8 \\
8 \\
0\end{array}$ & $\begin{array}{l}8 \\
8 \\
0\end{array}$ & $\begin{array}{l}8 \\
8 \\
0\end{array}$ \\
\hline & 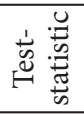 & $\begin{array}{l}\not{\infty} \\
\stackrel{\infty}{1}\end{array}$ & $\stackrel{n}{\stackrel{2}{m}}$ & $\begin{array}{l}\text { aे } \\
\text { ते }\end{array}$ & $\begin{array}{l}\stackrel{2}{1} \\
i n \\
n\end{array}$ & & 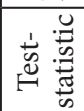 & 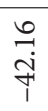 & $\begin{array}{l}\hat{N} \\
\hat{\mathrm{n}}\end{array}$ & $\begin{array}{l}\text { N. } \\
\stackrel{0}{0} \\
\infty \\
-1\end{array}$ & $\begin{array}{l}\infty \\
\stackrel{0}{n} \\
\infty \\
\infty \\
\infty\end{array}$ \\
\hline \multirow{2}{*}{ 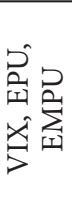 } & 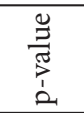 & $\begin{array}{l}8 \\
8 \\
\circ\end{array}$ & $\begin{array}{l}8 \\
8 \\
0\end{array}$ & $\begin{array}{l}8 \\
8 \\
0\end{array}$ & $\begin{array}{l}8 \\
8 \\
0\end{array}$ & \multirow{2}{*}{ 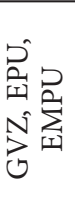 } & 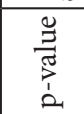 & $\begin{array}{l}8 \\
8 \\
0\end{array}$ & $\begin{array}{l}8 \\
8 \\
0\end{array}$ & $\begin{array}{l}8 \\
8 \\
0\end{array}$ & $\begin{array}{l}8 \\
8 \\
0\end{array}$ \\
\hline & 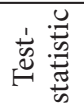 & $\underset{+}{\stackrel{\sim}{\sim}}$ & $\begin{array}{l}\underset{1}{\infty} \\
\stackrel{\infty}{T}\end{array}$ & $\begin{array}{l}\stackrel{0}{\leftrightarrows} \\
\stackrel{\sim}{\pi}\end{array}$ & $\begin{array}{l}\text { ஸे } \\
\text { in } \\
\text { ñ }\end{array}$ & & 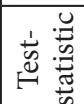 & 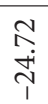 & $\stackrel{\widehat{N}}{\vec{m}}$ & 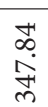 & ఏ్t \\
\hline $\begin{array}{l}\frac{0}{0} \\
\frac{0}{0} \\
\sum\end{array}$ & 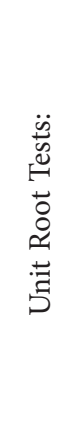 & 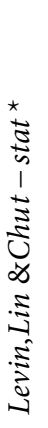 & 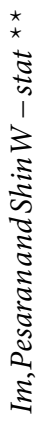 & 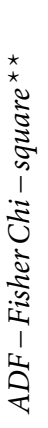 & 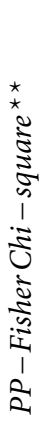 & $\begin{array}{l}\frac{0}{0} \\
\frac{1}{0} \\
\stackrel{0}{2}\end{array}$ &  & 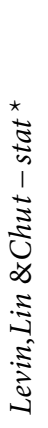 & 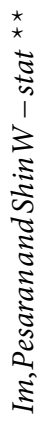 & 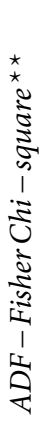 & 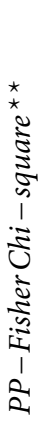 \\
\hline
\end{tabular}




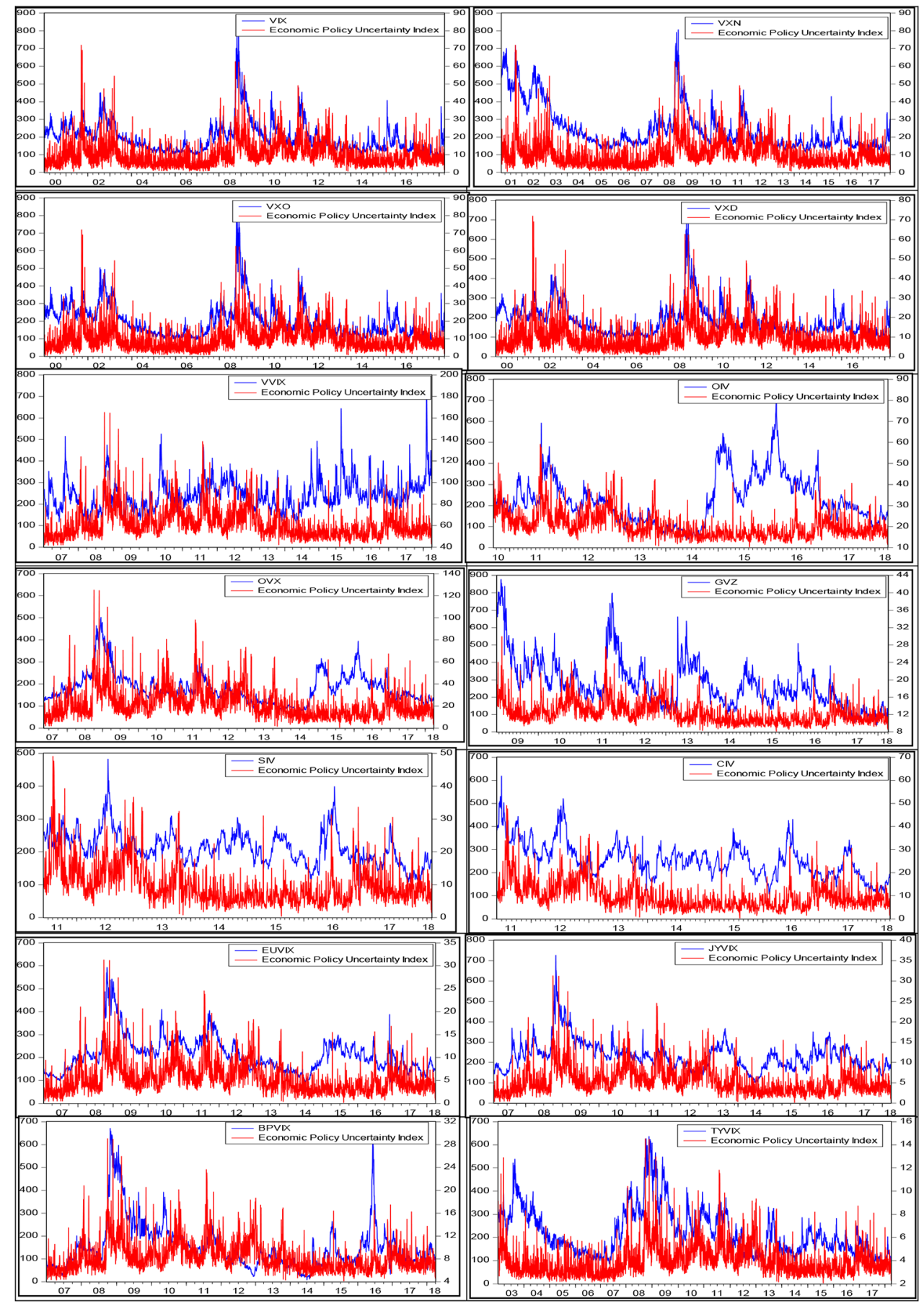

Figure 1. Time series plot of Implied volatility index across assets and Policy Uncertainty in the U.S. 


\section{Results and discussions ${ }^{2}$}

\subsection{Policy uncertainty and non-announcement period}

A non-announcement period is a period with the full amount of uncertainty concerning the economic outlook of the US economy. Due to the lack of information about the future states of nature, investors' anxiety goes at an extreme level. Such condition results in overburden on the hedge funds. Till the information is received, available investors keep buying options at a higher premium, particularly put options (e.g., Christensen \& Prabhala, 1998; Pástor \& Veronesi, 2013). Ultimately, this rally results in higher options' prices and a high level of implied volatility. Now looking at the intercept coefficient through Tables $4-8$, it is visible that equity market volatility remains positive during the non-announcement period. The positive statistically significant coefficient ranges from $5 \%$ to $21 \%$ imply that policy uncertainty and lack of information about future macroeconomic outcomes cause a substantial increase in the future stock market volatility. Moreover, similar evidence is obtained for the commodity market, which appears from $5 \%$ to $18 \%$. The policy uncertainty affects more the crude oil market and, secondly, the gold market. Corn and soybean markets also respond positively during market uncertainty. Additionally, currency markets such as for Euro, JPY, and BP also remain sensitive around market ambiguity in the FX rates. On average, the FX rate volatility appears to be $4 \%$ towards the positive side, followed by policy uncertainty. Lastly, the interest rate volatility does not escape from future uncertainty about the fed's rate changes.

Table 4. Economic policy uncertainty and the implied volatility index (VIX)

\begin{tabular}{|l|c|c|c|}
\hline \multicolumn{1}{|c|}{ Regressors } & $\mathrm{M} / \mathrm{V}$ & Estimate & Z-stat \\
\hline Intercept & $\mathrm{M}$ & $0.1106 \S$ & $9.88^{\mathrm{a}}$ \\
\hline FOMC & $\mathrm{M}$ & $-0.1218 \S$ & $-2.30^{\mathrm{b}}$ \\
\hline GDP & $\mathrm{M}$ & $-0.0155 \S$ & -0.52 \\
\hline MACRO & $\mathrm{M}$ & $-0.1612 \S$ & $-9.21^{\mathrm{a}}$ \\
\hline RSPX & $\mathrm{M}$ & $-97.5425 \S$ & $-113.44^{\mathrm{a}}$ \\
\hline AR(1) & $\mathrm{M}$ & -0.0772 & $-4.15^{\mathrm{a}}$ \\
\hline Intercept & $\mathrm{V}$ & 0.0481 & $6.61^{\mathrm{a}}$ \\
\hline ARCH & $\mathrm{V}$ & 0.2818 & $30.78^{\mathrm{a}}$ \\
\hline GARCH & $\mathrm{V}$ & 0.5994 & $48.19^{\mathrm{a}}$ \\
\hline EPU & $\mathrm{V}$ & $0.0191 \S$ & $2.28^{\mathrm{b}}$ \\
\hline EMPU & $\mathrm{V}$ & $0.0905 \S$ & $11.31^{\mathrm{a}}$ \\
\hline 2000s boom & $\mathrm{V}$ & -0.0464 & $-9.10^{\mathrm{a}}$ \\
\hline Early credit crunch & $\mathrm{V}$ & $0.0111 \S$ & 0.86 \\
\hline Lehman collapse \& recession & $\mathrm{V}$ & $0.1261 \S$ & $3.76^{\mathrm{a}}$ \\
\hline
\end{tabular}

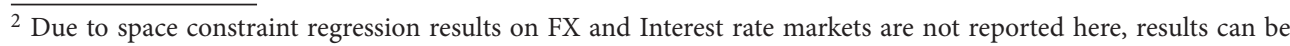
available on request.
} 
End of Table 4

\begin{tabular}{|c|c|c|c|}
\hline Regressors & $\mathrm{M} / \mathrm{V}$ & Estimate & Z-stat \\
\hline Fiscal policybattles & $\mathrm{V}$ & $0.0060 \$$ & 0.74 \\
\hline P.E.Year 2000Q4 & $\mathrm{V}$ & $0.0137 \S$ & 0.30 \\
\hline P.E.Year 2004Q4 & $\mathrm{V}$ & $0.0253 \S$ & 1.09 \\
\hline P.E.Year 2008Q4 & $\mathrm{V}$ & $0.8192 \$$ & $2.05^{b}$ \\
\hline P.E.Year 2012Q4 & $\mathrm{V}$ & -0.0436 & -1.45 \\
\hline P.E.Year 2016Q4 & $\mathrm{V}$ & $0.0019 \$$ & 0.09 \\
\hline Joint effects & & F-stat & p-value \\
\hline $\begin{array}{l}\text { Null Ho: FOMC = } \\
G D P=\text { Macro }=0\end{array}$ & & $31.73^{\mathrm{a}}$ & 0.000 \\
\hline $\begin{array}{l}\text { Null Ho: } \\
\text { P.E Year } 2000=2004=2008= \\
2012=2016=0\end{array}$ & & 1.47 & 0.196 \\
\hline Adj.R-squared & & 0.66 & \\
\hline$D W-$ stat & & 2.04 & \\
\hline $\log L$ & & -5156.517 & \\
\hline
\end{tabular}

Note: $₫$ signifies estimates appear as hypothesized in the regression model; Significant at ${ }^{\mathrm{a}} 1 \%,{ }^{\mathrm{b}} 5 \%$, ${ }^{c} 10 \%$ level.

\subsection{FOMC meeting day}

The present study considers 147 FOMC meeting days to examine what FOMC statements contain the information to explain the financial market. Analysts, investors, and volatility traders closely monitor the outcome of FOMC statements. By convention, once the FOMC statement released information enters the market and volatility goes at its normal level (e.g., Nikkinen et al., 2006). The coefficient on the FOMC variable appears to be negative (see Tables 4-8), and it is statistically significant. For example, VIX is the leading indicator of equity market valuation in the USA; on FOMC meeting day, the slope coefficient calculated -0.1218 and statistically significant at 5\% level. And it is true for VXN-, VXD- and VVIXbased implied volatility index. Tables 9 and 10 present the slopes on FOMC meeting day for OIV and OVX crude oil volatility indices. We can see that the slope appears to be negative but not statistically significant. It signifies that monetary policy uncertainty does not contain any information to explain crude oil price volatility, but as per a priori hypothesis, the slope appears negative. Apriori hypothesis indicates that FOMC meeting day matters for gold market participants; thus, gold market volatility falls on FOMC day, as shown in Table 11. On the other hand, commodities such as corn and soybean do not respond significantly. Does monetary policy uncertainty affect the exchange rate volatility? The answer is yes, the empirical outcome shows that the Euro-USD volatility index falls considerably on the FOMC meeting day. At the same time, JPY-USD and BP-USD do not respond aggressively. Moreover, the US Treasury note volatility index has responded significantly with negative estimates. It also implies that FOMC meeting day matters for the interest rates in the US money market. 
Table 5. Economic policy uncertainty and Nasdaq volatility index (VXN)

\begin{tabular}{|c|c|c|c|}
\hline Regressors & $\mathrm{M} / \mathrm{V}$ & Estimate & Z-stat \\
\hline Intercept & $M$ & $0.1568 \S$ & $10.64^{\mathrm{a}}$ \\
\hline FOMC & M & $-0.1598 \S$ & $-2.76^{\mathrm{a}}$ \\
\hline$G D P$ & M & $-0.0293 \S$ & -0.56 \\
\hline MACRO & M & $-0.2218 \$$ & $-9.86^{\mathrm{a}}$ \\
\hline$R N A S D A Q$ & M & $-79.8846 \$$ & $-91.44^{\mathrm{a}}$ \\
\hline$A R(1)$ & M & -0.0287 & $-1.68^{c}$ \\
\hline Intercept & $\mathrm{V}$ & 0.0216 & $2.86^{\mathrm{a}}$ \\
\hline$A R C H$ & $\mathrm{~V}$ & 0.1463 & $16.88^{\mathrm{a}}$ \\
\hline GARCH & $\mathrm{V}$ & 0.7458 & $64.44^{\mathrm{a}}$ \\
\hline$E P U$ & $\mathrm{~V}$ & $0.0225 \$$ & $2.30^{\mathrm{b}}$ \\
\hline$E M P U$ & $\mathrm{~V}$ & $0.1379 \$$ & $10.69^{\mathrm{a}}$ \\
\hline 2000sboom & $\mathrm{V}$ & -0.0256 & $-5.36^{\mathrm{a}}$ \\
\hline Earlycredit crunch & $\mathrm{V}$ & -0.0046 & -0.36 \\
\hline Lehman collapse \& recession & $\mathrm{V}$ & $0.0340 \$$ & $1.84^{\mathrm{c}}$ \\
\hline Fiscal policybattles & $\mathrm{V}$ & -0.0237 & $-4.25^{\mathrm{a}}$ \\
\hline P.E.Year 2004Q4 & $\mathrm{V}$ & -0.0037 & -0.23 \\
\hline P.E.Year 2008Q4 & $\mathrm{V}$ & $0.5567 \S$ & $1.99^{\mathrm{b}}$ \\
\hline P.E.Year 2012Q4 & $\mathrm{V}$ & -0.0592 & $-3.19^{a}$ \\
\hline P.E.Year 2016Q4 & $\mathrm{V}$ & -0.0404 & $-3.23^{a}$ \\
\hline Joint effects & & F-stat & $\mathrm{p}$-value \\
\hline $\begin{array}{l}\text { Null Ho: FOMC }=G D P= \\
\text { Macro }=0\end{array}$ & & 39.39 & 0.000 \\
\hline $\begin{array}{l}\text { Null Ho: P.E Year } 2004= \\
2008=2012=2016=0\end{array}$ & & 5.85 & 0.000 \\
\hline Adj.R-squared & & 0.55 & \\
\hline$D W-$ stat & & 2.00 & \\
\hline $\log L$ & & -5413.83 & \\
\hline
\end{tabular}

Note: $₫$ signifies estimates appear as hypothesized in the regression model; Significant at ${ }^{\mathrm{a}} 1 \%,{ }^{\mathrm{b}} 5 \%$, ${ }^{c} 10 \%$ level.

\subsection{GDP report}

The uncertainty of the GDP estimates significantly affects financial markets. Once the GDP data is available, all the volatility indices are supposed to fall and reach their normal level. On the GDP report day, Tables 4-8 explains that it does not affect the investors' sentiment significantly when looking at the variant of VIX for the equity market. In fact, on the GDP report day, volatility indices fall but not significantly. Tables 9 and 10 show the estimates on 
GDP report day specific to the oil market regarding OIV and OVX. The WTI futures market and GDP data show a significant negative impact, while OVX does not exhibit faster movement. It implies that GDP-related uncertainty causes energy prices in the US energy market, and OIV does contain future volatility of crude oil prices. Other commodities such as gold, corn, and soybean do not overreact subject to scheduled GDP report. On the release of the GDP report, the GVZ shows a marginal decrease in the volatility. Now moving to the FX rate volatility on the GDP report, again, there is no significant co-movement between USD and Euro-BP. At the same time, JPY-USD overreaction is higher at a $10 \%$ level. It also makes sense that JPY-USD is more sensitive to the US GDP report uncertainty. Lastly, the interest rate does not behave significantly, but there is a marginal increase in the interest rate volatility subject to the recent GDP announcement.

\subsection{Other macroeconomic indicators}

The US macroeconomic indicators such as "PPI, CPI, state employment, employment situations, labour turnover, and job openings, US export/imports, unemployment, real earnings, earnings of wages and salary, business employment dynamics and employment cost index" and their uncertainty play a significant role in the equity market and other markets. Tables 4-8 evidence that other macroeconomic indicators do contain substantial evidence to enlighten the equity market. The uncertainty with regard to macroeconomic news causes a substantial increase in the VIX level. When the outcomes are publicised, the ambiguity becomes resolved, and implied volatility reverts to its normal level. Most of the volatility indices fall significantly on the announcement of other scheduled macroeconomic indicators. Tables 9 and 10 show the OIV- and OVX-based crude oil price volatility index, and we can see that on the scheduled macroeconomic announcements, crude oil price volatility falls rapidly. It also indicates that uncertainty about the macroeconomy influences the future price of crude oil. Other commodities such as gold, corn, and soybean also exhibit a similar outcome as reported for the abovementioned markets. At this point, one can say that uncertainty about the macroeconomic data impacts commodity prices, and GVZ, SIV, and CIV are the best measures of investors' sentiment in the commodity market. The FX market volatility appears to be very high concerning FOMC and GDP news releases. The outcome implies that the US macroeconomic data matter for the exchange rate across the global currency market. At the outset, one can say that macroeconomic news releases contain information to influence interest rates in the US money market. On the day of the scheduled macroeconomic announcement, TYVIX falls significantly (see Tables 11-13).

Table 6. Economic policy uncertainty and OEX implied volatility index (VXO)

\begin{tabular}{|l|c|c|c|}
\hline \multicolumn{1}{|c|}{ Regressors } & $\mathrm{M} / \mathrm{V}$ & Estimate & Z-stat \\
\hline Intercept & $\mathrm{M}$ & $0.0543 \S$ & $4.22^{\mathrm{a}}$ \\
\hline FOMC & $\mathrm{M}$ & 0.0178 & 0.33 \\
\hline$G D P$ & $\mathrm{M}$ & 0.0552 & 1.39 \\
\hline
\end{tabular}


End of Table 6

\begin{tabular}{|c|c|c|c|}
\hline Regressors & $\mathrm{M} / \mathrm{V}$ & Estimate & Z-stat \\
\hline MACRO & $M$ & $-0.0467 \S$ & $-2.34^{\mathrm{a}}$ \\
\hline$R S P X 100$ & M & -114.3266 & $-119.56^{a}$ \\
\hline$A R(1)$ & M & -0.1069 & $-6.09^{a}$ \\
\hline Intercept & $\mathrm{V}$ & 0.0635 & $7.86^{\mathrm{a}}$ \\
\hline$A R C H$ & $\mathrm{~V}$ & 0.1892 & $16.33^{\mathrm{a}}$ \\
\hline GARCH & $\mathrm{V}$ & 0.6998 & $55.20^{\mathrm{a}}$ \\
\hline$E P U$ & $\mathrm{~V}$ & $0.0157 \S$ & $1.82^{\mathrm{c}}$ \\
\hline$E M P U$ & $\mathrm{~V}$ & $0.0825 \$$ & $10.32^{\mathrm{a}}$ \\
\hline 2000sboom & $\mathrm{V}$ & -0.0590 & $-10.00^{\mathrm{a}}$ \\
\hline Earlycredit crunch & $\mathrm{V}$ & $0.0029 \$$ & 0.18 \\
\hline Lehmancollapse \& recession & $\mathrm{V}$ & $0.0818 \S$ & $3.04^{\mathrm{a}}$ \\
\hline Fiscal policybattles & $\mathrm{V}$ & -0.0339 & $-4.65^{a}$ \\
\hline P.E.Year 2000Q4 & V & -0.0142 & -0.30 \\
\hline P.E.Year 2004Q4 & $\mathrm{V}$ & $0.0387 \S$ & $1.70^{\mathrm{c}}$ \\
\hline P.E.Year 2008Q4 & $\mathrm{V}$ & $1.2708 \$$ & $2.20^{\mathrm{b}}$ \\
\hline P.E.Year 2012Q4 & $\mathrm{V}$ & -0.0246 & -1.04 \\
\hline P.E.Year 2016Q4 & $\mathrm{V}$ & -0.0001 & 0.01 \\
\hline Joint effects & & F-stat & p-value \\
\hline $\begin{array}{l}\text { Null Ho: } F O M C=G D P= \\
\text { Macro }=0\end{array}$ & & $2.36^{\mathrm{a}}$ & 0.0687 \\
\hline $\begin{array}{l}\text { Null Ho: P.E Year } 2000=2004= \\
2008=2012=2016=0\end{array}$ & & $1.73^{c}$ & 0.1221 \\
\hline Adj.R-squared & & 0.68 & \\
\hline DW-stat & & 2.12 & \\
\hline $\log L$ & & -5621.318 & \\
\hline
\end{tabular}

Note: $\S$ signifies estimates appear as hypothesized in the regression model; Significant at ${ }^{\mathrm{a}} 1 \%,{ }^{\mathrm{b}} 5 \%$, ${ }^{c} 10 \%$ level.

\subsection{Effects of policy uncertainty on the implied volatility index}

Baker et al. (2016) develop and disseminate the policy-uncertainty-related index known as the EPU index. The EPU index is the overall measure of the uncertainty about the US economy. Its values are taken as an exogenous factor in the variance equation to model the volatility of major volatility indices. The estimates on the EPU index through Tables $4-8$ seem to be encouraging and statistically significant. The result indicates that EPU causes positively to the investors' sentiment. Also, the equity-market-specific policy uncertainty causes higher 
on the counterpart of EPU-specific uncertainty. The estimates across equity market EMPU VIX appears to be higher as compared to EPU estimates. The EPU and EMPU indices are the measures of uncertainty, and they should cause to increase in the volatility when it spikes. The empirical outcome supports the stated a priori hypothesis that policy uncertainty causes an increase in the markets' expected volatility. The effects of EPU on the crude oil market is found to be negative, while the equity-market-specific EMPU shows positive effects on the future price of the crude oil market. Now, looking at the gold market, volatility tends to be positive concerning EMPU uncertainty, while commodities such as corn and soybean have shown a mixed outcome. One of the essential findings from Tables 12 and 13 is that commodities' prices remain calm in anticipation of policy uncertainty. Now analysing the FX market, policy uncertainty, and exchange rate across different currencies and such effects are asymmetric. Euro-USD shows adverse effects, and JPY-USD is negative for EPU and positive in response to EMPU. A similar pattern has also been reported for the domestic interest rates gauged into TYVIX.

\subsection{Effects of political and economic events on the VIX}

The study also considers four major economic and political events that took place from 2000 to 2018. (i) "2000s commodity boom" is one of the important events that took place from January 2003 to June 2007, and its influence on the equity market seems adverse and significant. The negative coefficient concerning VIX throughout this period advocates that commodity-related flourishing has instigated equity market volatility to tumble till June 2007. Moreover, other asset classes, such as crude oil and T-bill, also revealed comparable effects. (ii) The "credit crunch" has been observed through July 2007 to August 2008, and it is the period of the credit turmoil, and it has expressively hindered the global banking system over an upsurge in the interest rates. The crude oil prices affected adversely while the equity market remained unaffected on the eve of the credit crunch. Moreover, the short-term interest rate and foreign exchange market volatility have been increased following the credit crunch. Additionally, during the credit turmoil period, USD exchange rate volatility concerning major currencies Euro, Japanese Yen, and British Pound appears to be calm, while interest rate volatility remains higher. (iii) Lehman's collapse and the global recession happened from September 2008 to December 2009, the volatility across various asset class and investors overreaction found to be at an extreme level. (iv) The "fiscal policy cliff" the period that extended from January 2010 to October 2013, the amalgamation of five taxes rises and two expenditure cuts. There was a substantial amount of uncertainty predominant in this period concerning expenditure and government taxes, and that might have caused in one more financial disaster. Fiscal clashes do not enlighten the stock market volatility expressively, while the crude oil market has obstructed unfavourably. The uncertainty associated with the fiscal regime affected the commodities markets such as crude oil, gold, corn, and soybean significantly. Furthermore, short term interest rate volatility has also stimulated adversely (the results are evident from Tables 4-13). 
Table 7. Economic policy uncertainty and Dow Jon volatility index (VXD)

\begin{tabular}{|c|c|c|c|}
\hline Regressors & $\mathrm{M} / \mathrm{V}$ & Estimate & Z-stat \\
\hline Intercept & $\mathrm{M}$ & $0.0943 \$$ & $7.68^{a}$ \\
\hline FOMC & $\mathrm{M}$ & -0.1734 & $-3.15^{\mathrm{a}}$ \\
\hline GDP & M & $0.0200 \$$ & 0.52 \\
\hline$M A C R O$ & M & $-0.1502 \$$ & $-7.89^{\mathrm{a}}$ \\
\hline$R D J I$ & M & -84.6950 & $-114.09^{a}$ \\
\hline$A R(1)$ & M & -0.1110 & $-6.20^{\mathrm{a}}$ \\
\hline Intercept & $\mathrm{V}$ & 0.0451 & $8.45^{\mathrm{a}}$ \\
\hline$A R C H$ & V & 0.2351 & $18.40^{\mathrm{a}}$ \\
\hline GARCH & $\mathrm{V}$ & 0.6321 & $51.61^{\mathrm{a}}$ \\
\hline$E P U$ & V & $0.0108 \$$ & 1.33 \\
\hline$E M P U$ & V & $0.0765 \$$ & $11.18^{\mathrm{a}}$ \\
\hline 2000 sboom & $\mathrm{V}$ & -0.0188 & $-5.53^{\mathrm{a}}$ \\
\hline Early credit crunch & $\mathrm{V}$ & $0.0212 \$$ & 1.55 \\
\hline Lehmancollapse \& recession & $\mathrm{V}$ & $0.0957 \$$ & $3.99^{\mathrm{a}}$ \\
\hline Fiscal policy battles & $\mathrm{V}$ & $0.0064 \S$ & 0.89 \\
\hline P.E.Year 2000Q4 & V & $0.0319 \$$ & 1.00 \\
\hline P.E.Year 2004Q4 & V & $0.0073 \S$ & 0.33 \\
\hline P.E.Year 2008Q4 & $\mathrm{V}$ & $0.8368 \$$ & $1.95^{c}$ \\
\hline P.E.Year 2012Q4 & V & $0.2168 \$$ & $8.10^{\mathrm{a}}$ \\
\hline P.E.Year 2016Q4 & V & -0.0168 & -0.93 \\
\hline Joint effects & & F-stat & $\mathrm{p}$-value \\
\hline $\begin{array}{l}\text { Null Ho: FOMC }=G D P= \\
\text { Macro }=0\end{array}$ & & $24.85^{\mathrm{a}}$ & 0.000 \\
\hline $\begin{array}{l}\text { Null Ho: P.E Year } 2004= \\
2008=2012=2016=0\end{array}$ & & $14.16^{\mathrm{a}}$ & 0.000 \\
\hline Adj.R-squared & & 0.58 & \\
\hline DW-stat & & 2.10 & \\
\hline $\log L$ & & -5095.24 & \\
\hline
\end{tabular}

Note: $₫$ signifies estimates appear as hypothesized in the regression model; Significant at ${ }^{\mathrm{a}} 1 \%,{ }^{\mathrm{b}} 5 \%$, ${ }^{\mathrm{c}} 10 \%$ level.

\subsection{Presidential election year}

Does the presidential election contain some evidence to describe markets' future volatility (VIX)? The answer is yes. Election Year is the year with the higher amount of uncertainty. More specifically, the fourth quarter of the election year shows a more considerable amount of fluctuation in the stock prices and other commodity prices. The present study considers five presidential election years with dummies denoted by Q4 (fourth quarter of the election 
year). The reason this is the period of election poll an announcement of next presidents-elect of the U.S. The empirical outcome evidence that presidential elections have significantly influenced the equity market and commodity market as well. The joint hypothesis tests based on Wald F-statistics evidence that presidential polls create more market uncertainty and ultimately results in a rise of expected stock market volatility; other markets also show similar effects. It has been apparent from Table 10 that the presidential election year 2008Q4 and 2016Q4 have caused a significant increase in the oil market volatility, and it is also true for the corn and soybean markets. The exchange rate also remained more volatile concerning the election-year uncertainty. The interest rate volatility also exhibited positive during the fourth quarter of the election years 2004 and 2008.

Table 8. Economic policy uncertainty and VVIX

\begin{tabular}{|c|c|c|c|}
\hline Regressors & $\mathrm{M} / \mathrm{V}$ & Estimate & Z-stat \\
\hline Intercept & M & $0.2101 \S$ & $2.12^{b}$ \\
\hline FOMC & M & $-0.5415 \S$ & $-1.69^{c}$ \\
\hline$G D P$ & M & $-0.3477 \S$ & -1.18 \\
\hline MACRO & M & 0.0183 & 0.14 \\
\hline$R S P X$ & M & -237.1064 & $-41.45^{\mathrm{a}}$ \\
\hline$A R(1)$ & M & -0.0441 & $-2.54^{b}$ \\
\hline Intercept & $\mathrm{V}$ & 4.0776 & $9.77^{\mathrm{a}}$ \\
\hline $\mathrm{ARCH}$ & $\mathrm{V}$ & 0.2202 & $15.08^{\mathrm{a}}$ \\
\hline GARCH & $\mathrm{V}$ & 0.4407 & $14.21^{\mathrm{a}}$ \\
\hline$E P U$ & $\mathrm{~V}$ & 0.9716 & $2.99^{\mathrm{a}}$ \\
\hline$E M P U$ & V & 4.6676 & $11.60^{\mathrm{a}}$ \\
\hline Early credit crunch & V & -2.4684 & $-4.50^{\mathrm{a}}$ \\
\hline Lehmancollapse \& recession & V & -1.4635 & $-2.44^{\mathrm{v}}$ \\
\hline Fiscal policybattles & V & -3.1159 & $-9.42^{\mathrm{a}}$ \\
\hline P.E.Year 2008Q4 & $\mathrm{V}$ & 13.8121 & $2.20^{\mathrm{b}}$ \\
\hline P.E.Year 2012Q4 & V & -0.9679 & -1.17 \\
\hline P.E.Year 2016Q4 & V & -1.7217 & $-1.96^{\mathrm{b}}$ \\
\hline Joint effects & & F-stat & p-value \\
\hline $\begin{array}{l}\text { Null Ho: FOMC }=G D P= \\
\text { Macro }=0\end{array}$ & & 1.48 & 0.218 \\
\hline $\begin{array}{l}\text { Null Ho: P.E Year } 2008= \\
2012=2016=0\end{array}$ & & $3.23^{\mathrm{a}}$ & 0.022 \\
\hline Adj.R-squared & & 0.30 & \\
\hline$D W-s t a t$ & & 2.00 & \\
\hline $\log L$ & & -7672.70 & \\
\hline
\end{tabular}

Note: $₫$ signifies estimates appear as hypothesized in the regression model; Significant at ${ }^{\mathrm{a}} 1 \%$, ${ }^{\mathrm{b}} 5 \%$, ${ }^{c} 10 \%$ level. 
Table 9. Economic policy uncertainty and WTI oil volatility index (OIV)

\begin{tabular}{|c|c|c|c|}
\hline Regressors & $\mathrm{M} / \mathrm{V}$ & Estimate & Z-stat \\
\hline Intercept & M & $0.1840 \$$ & $4.81^{\mathrm{a}}$ \\
\hline FOMC & M & $-0.1083 \S$ & -0.67 \\
\hline GDP & M & $-0.2472 \S$ & $-1.80^{c}$ \\
\hline MACRO & M & $-0.3618 \S$ & $-6.81^{\mathrm{a}}$ \\
\hline$R W T I$ & M & -34.6280 & $-30.20^{a}$ \\
\hline$A R(1)$ & M & -0.0082 & -0.32 \\
\hline Intercept & $\mathrm{V}$ & 0.1735 & $6.82^{\mathrm{a}}$ \\
\hline$A R C H$ & $\mathrm{~V}$ & 0.1620 & $14.50^{\mathrm{a}}$ \\
\hline GARCH & $\mathrm{V}$ & 0.7838 & $56.93^{\mathrm{a}}$ \\
\hline$E P U$ & $\mathrm{~V}$ & -0.1180 & $-4.28^{a}$ \\
\hline$E M P U$ & $\mathrm{~V}$ & $0.2135 \$$ & $6.42^{\mathrm{a}}$ \\
\hline Fiscal policybattles & $\mathrm{V}$ & $0.0604 \S$ & $3.24^{\mathrm{a}}$ \\
\hline P.E.Year 2012Q4 & $\mathrm{V}$ & -0.0407 & -1.04 \\
\hline P.E.Year 2016Q4 & $\mathrm{V}$ & $0.0996 \$$ & $1.65^{c}$ \\
\hline Joint effects & & F-stat & $\mathrm{p}$-value \\
\hline Null Ho: FOMC $=$ GDP $=$ Macro $=0$ & & $20.55^{\mathrm{a}}$ & 0.000 \\
\hline Null Ho: P.E Year $2012=2016=0$ & & $1.86^{\mathrm{c}}$ & 0.156 \\
\hline Adj.R-squared & & 0.19 & \\
\hline DW-stat & & 2.04 & \\
\hline $\log L$ & & -3265.46 & \\
\hline
\end{tabular}

Note: $₫$ signifies estimates appear as hypothesized in the regression model; Significant at ${ }^{\mathrm{a}} 1 \%,{ }^{\mathrm{b}} 5 \%$, ${ }^{c} 10 \%$ level.

Table 10. Economic policy uncertainty and USO ETF oil volatility index (OVX)

\begin{tabular}{|l|c|c|c|}
\hline \multicolumn{1}{|c|}{ Regressors } & $\mathrm{M} / \mathrm{V}$ & Estimate & Z-stat \\
\hline Intercept & $\mathrm{M}$ & $0.1340 \S$ & $3.78^{\mathrm{a}}$ \\
\hline FOMC & $\mathrm{M}$ & $-0.0748 \S$ & -0.48 \\
\hline GDP & $\mathrm{M}$ & 0.0994 & 0.87 \\
\hline MACRO & $\mathrm{M}$ & $-0.2783 \S$ & $-5.48^{\mathrm{a}}$ \\
\hline RUSO & $\mathrm{M}$ & -35.1541 & $-36.96^{\mathrm{a}}$ \\
\hline AR(1) & $\mathrm{M}$ & -0.0262 & -1.21 \\
\hline Intercept & $\mathrm{V}$ & 0.0970 & $7.19^{\mathrm{a}}$ \\
\hline ARCH & $\mathrm{V}$ & 0.1276 & $11.75^{\mathrm{a}}$ \\
\hline GARCH & $\mathrm{V}$ & 0.8290 & $80.71^{\mathrm{a}}$ \\
\hline EPU & $\mathrm{V}$ & -0.0590 & $-3.59^{\mathrm{a}}$ \\
\hline
\end{tabular}


End of Table 10

\begin{tabular}{|c|c|c|c|}
\hline Regressors & $\mathrm{M} / \mathrm{V}$ & Estimate & Z-stat \\
\hline$E M P U$ & $\mathrm{~V}$ & $0.1725 \$$ & $6.95^{\mathrm{a}}$ \\
\hline 2000sboom & $\mathrm{V}$ & -0.1297 & $-2.17^{b}$ \\
\hline Earlycredit crunch & $\mathrm{V}$ & $0.1151 \S$ & $6.40^{a}$ \\
\hline Lehmancollapse \& recession & $\mathrm{V}$ & $0.0932 \S$ & $4.04^{\mathrm{a}}$ \\
\hline Fiscal policy battles & $\mathrm{V}$ & $0.0530 \$$ & $4.73^{\mathrm{a}}$ \\
\hline P.E.Year $2008 Q 4$ & $\mathrm{~V}$ & $1.7106 \$$ & $2.18^{\mathrm{b}}$ \\
\hline P.E.Year 2012Q4 & $\mathrm{V}$ & -0.0858 & $-4.93^{b}$ \\
\hline P.E.Year 2016Q4 & V & $0.1133 \$$ & 1.61 \\
\hline Joint effects & & F-stat & p-value \\
\hline Null Ho: FOMC $=G D P=$ Macro $=0$ & & $10.60^{\mathrm{a}}$ & 0.000 \\
\hline $\begin{array}{l}\text { Null Ho: P.E Year } 2008=2012= \\
2016=0\end{array}$ & & $9.64^{\mathrm{a}}$ & 0.000 \\
\hline Adj.R-squared & & 0.17 & \\
\hline DW-stat & & 2.12 & \\
\hline $\log L$ & & -4918.97 & \\
\hline
\end{tabular}

Note: $₫$ signifies estimates appear as hypothesized in the regression model; Significant at ${ }^{\mathrm{a}} 1 \%,{ }^{\mathrm{b}} 5 \%$, ${ }^{c} 10 \%$ level.

Table 11. Economic policy uncertainty and SPDR gold volatility index (GVZ)

\begin{tabular}{|l|c|c|c|}
\hline \multicolumn{1}{|c|}{ Regressors } & $\mathrm{M} / \mathrm{V}$ & Estimate & Z-stat \\
\hline Intercept & $\mathrm{M}$ & $0.0952 \S$ & $3.63^{\mathrm{a}}$ \\
\hline FOMC & $\mathrm{M}$ & $-0.2851 \S$ & $-2.64^{\mathrm{b}}$ \\
\hline GDP & $\mathrm{M}$ & $-0.1079 \S$ & -1.26 \\
\hline MACRO & $\mathrm{M}$ & $-0.1547 \S$ & $-4.28^{\mathrm{a}}$ \\
\hline RSPDR & $\mathrm{M}$ & -9.0230 & $-6.74^{\mathrm{a}}$ \\
\hline AR(1) & $\mathrm{M}$ & -0.0578 & $-2.49^{\mathrm{b}}$ \\
\hline Intercept & $\mathrm{V}$ & 0.0752 & $6.37^{\mathrm{a}}$ \\
\hline ARCH & $\mathrm{V}$ & 0.1658 & $15.38^{\mathrm{a}}$ \\
\hline GARCH & $\mathrm{V}$ & 0.7339 & $46.09^{\mathrm{a}}$ \\
\hline EPU & $\mathrm{V}$ & -0.0585 & $-3.99^{\mathrm{a}}$ \\
\hline EMPU & $\mathrm{V}$ & $0.1812 \S$ & $7.12^{\mathrm{a}}$ \\
\hline Lehmancollapse \& recession & $\mathrm{V}$ & $0.2426 \S$ & $6.25^{\mathrm{a}}$ \\
\hline Fiscal policybattles & $\mathrm{V}$ & $0.0813 \S$ & $6.20^{\mathrm{a}}$ \\
\hline P.E.Year 2012Q4 & $\mathrm{V}$ & -0.0239 & -1.02 \\
\hline P.E.Year 2016Q4 & $\mathrm{V}$ & $0.0247 \S$ & 0.83 \\
\hline
\end{tabular}


End of Table 11

\begin{tabular}{|l|c|c|c|}
\hline \multicolumn{1}{|c|}{ Regressors } & M/V & Estimate & Z-stat \\
\hline Joint effects & & F-stat & p-value \\
\hline Null Ho: FOMC $=$ GDP $=$ Macro $=0$ & & $6.80^{\text {a }}$ & 0.0001 \\
\hline Null Ho: P.E Year 2012 = 2016=0 & & 0.87 & 0.4182 \\
\hline Adj.R-squared & & 0.03 & \\
\hline DW -stat & & 2.09 & \\
\hline Log L & & -3227.72 & \\
\hline
\end{tabular}

Note: $\S=$ signifies estimates appear as hypothesized in the regression model; Significant at ${ }^{\mathrm{a}} 1 \%$, ${ }^{\mathrm{b}} 5 \%$, ${ }^{\mathrm{c}} 10 \%$ level.

The combined effects of uncertainty of monetary policy, production and real economic activity, and other macroeconomic factors appear statistically significant. The significant Wald F-statistics through Tables 4-13 signifies substantial effects for equity, commodity, FX, and interest rates. These crucial findings imply that a market participant to various market specifics takes into regard jointly the information related to monetary policy, gross domestic product, and major economic indicators in financial planning and risk management.

Finally, one can say that uncertainty (e.g., EPU, EMPU) and other macroeconomic indicators have shown profound effects on the volatility of several market specifics. The $A R C H$ and GARCH parameters appears positive across all asset classes. The results also signify that volatility persists concerning policy uncertainty for equity, commodity, and short-term interest rates. Demonstrating the volatility of the volatility index (VIX) provides insights that policy uncertainty encompasses essential market-vide evidence to describe the markets' expected volatility. Moreover, Baker et al. (2019) further develop the text-based equity market volatility (EMV) index, popularly known as policy news and stock market volatility indices. The EMV index can also be applied to examine the investors' sentiment across various asset classes.

Table 12. Economic policy uncertainty and corn implied volatility index (CIV)

\begin{tabular}{|l|c|c|c|}
\hline \multicolumn{1}{|c|}{ Regressors } & $\mathrm{M} / \mathrm{V}$ & Estimate & Z-stat \\
\hline Intercept & $\mathrm{M}$ & $0.0540 \S$ & 1.45 \\
\hline FOMC & $\mathrm{M}$ & 0.2204 & 1.09 \\
\hline$G D P$ & $\mathrm{M}$ & 0.1018 & 1.04 \\
\hline MACRO & $\mathrm{M}$ & $-0.1770 \S$ & $-3.41^{\mathrm{a}}$ \\
\hline AR(1) & $\mathrm{M}$ & -0.0314 & -1.23 \\
\hline Intercept & $\mathrm{V}$ & 0.0924 & $6.23^{\mathrm{a}}$ \\
\hline ARCH & $\mathrm{V}$ & 0.0959 & $9.87^{\mathrm{a}}$ \\
\hline GARCH & $\mathrm{V}$ & 0.8459 & $69.42^{\mathrm{a}}$ \\
\hline EPU & $\mathrm{V}$ & -0.0451 & $-2.89^{\mathrm{a}}$ \\
\hline EMPU & $\mathrm{V}$ & $0.0291 \S$ & $1.79^{\mathrm{c}}$ \\
\hline
\end{tabular}


End of Table 12

\begin{tabular}{|l|c|c|c|}
\hline \multicolumn{1}{|c|}{ Regressors } & $\mathrm{M} / \mathrm{V}$ & Estimate & Z-stat \\
\hline Fiscal policybattles & $\mathrm{V}$ & 0.0664 & $4.72^{\mathrm{a}}$ \\
\hline P.E.Year 2012Q4 & $\mathrm{V}$ & -0.0044 & -0.14 \\
\hline P.E.Year 2016Q4 & $\mathrm{V}$ & 0.2963 & $8.43^{\text {a }}$ \\
\hline Joint effects & & F-stat & p-value \\
\hline Null Ho: FOMC $=$ GDP = Macro $=0$ & & 1.59 & 0.190 \\
\hline Null Ho: P.E Year 2012=2016=0 & & $35.57^{\text {a }}$ & 0.000 \\
\hline Adj.R-squared & & 0.02 & \\
\hline DW-stat & & 2.09 & \\
\hline Log L & & -2620.92 & \\
\hline
\end{tabular}

Note: $₫$ signifies estimates appear as hypothesized in the regression model; Significant at ${ }^{\mathrm{a}} 1 \%,{ }^{\mathrm{b}} 5 \%$, ${ }^{\mathrm{c}} 10 \%$ level.

Table 13. Economic policy uncertainty and soybean implied volatility index (SIV)

\begin{tabular}{|l|c|c|c|}
\hline \multicolumn{1}{|c|}{ Regressors } & $\mathrm{M} / \mathrm{V}$ & Estimate & Z-stat \\
\hline Intercept & $\mathrm{M}$ & $0.1451 \S$ & $2.90^{\mathrm{a}}$ \\
\hline FOMC & $\mathrm{M}$ & $-0.0860 \S$ & -0.21 \\
\hline GDP & $\mathrm{M}$ & 0.0566 & 0.31 \\
\hline MACRO & $\mathrm{M}$ & $-0.2422 \S$ & $-3.47^{\mathrm{a}}$ \\
\hline AR(1) & $\mathrm{M}$ & -0.0441 & -1.29 \\
\hline Intercept & $\mathrm{V}$ & 1.7135 & $14.62^{\mathrm{a}}$ \\
\hline ARCH & $\mathrm{V}$ & 0.2324 & $9.08^{\mathrm{a}}$ \\
\hline GARCH & $\mathrm{V}$ & 0.2215 & $4.02^{\mathrm{a}}$ \\
\hline EPU & $\mathrm{V}$ & -0.1517 & -2.04 \\
\hline EMPU & $\mathrm{V}$ & -0.1680 & $-5.33^{\mathrm{a}}$ \\
\hline Fiscal policybattles & $\mathrm{V}$ & $0.9656 \S$ & $10.44^{\mathrm{a}}$ \\
\hline P.E.Year 2012Q4 & $\mathrm{V}$ & -0.9548 & $-4.30^{\mathrm{a}}$ \\
\hline P.E.Year 2016Q4 & $\mathrm{V}$ & -0.9417 & $-8.49^{\mathrm{a}}$ \\
\hline Joint effects & & $\mathrm{F}-\mathrm{stat}$ & $\mathrm{p}^{\mathrm{v}} \mathrm{value}$ \\
\hline Null Ho: FOMC $=$ GDP $=$ Macro $=0$ & & $4.12^{\mathrm{a}}$ & 0.006 \\
\hline Null Ho: P.E Year 2012 $2016=0$ & & $86.95^{\mathrm{a}}$ & 0.000 \\
\hline Adj.R-squared & & 0.02 & \\
\hline DW - stat & & 2.35 & \\
\hline Log L & & -3189.68 & \\
\hline Note: S signifs estim & & & \\
\hline
\end{tabular}

Note: $₫$ signifies estimates appear as hypothesized in the regression model; Significant at ${ }^{\mathrm{a}} 1 \%,{ }^{\mathrm{b}} 5 \%$, ${ }^{\mathrm{c}} 10 \%$ level. 


\section{Conclusions}

Does policy uncertainty contain important information to explain equity, commodity, and FX rates? The answer is yes. To discover the effects of such policy uncertainty on the various assets class, we consider 14 different volatility indices of the US market. The study analyses the equity, commodity, exchange rate, and interest rates for the sample period January 2000-March 2018. Policy uncertainty indices such as EPU and EMPU considered examining the influence of policy uncertainty on the various market volatility indices. Besides, for the empirical model-building, our study considers the FOMC report, GDP, and other macroeconomic indicators. Additionally, major political and economic events and US presidential election years have also been included in the empirical model to disclose the effects of policy uncertainty. The empirical model has expressed in terms of the ARCH and GARCHX framework.

The empirical outcomes evidence that market volatility remains high and positive during the economic uncertainty period. The lack of economic and political future movements raises economic uncertainty, and this uncertainty affects various market specifics. The study uncovers that the uncertainty of future events has gauged into VIX-based volatility measures across different markets. The future market position of an investor is subject to the uncertainty of FOMC, GDP, and macroeconomic data. Market participants jointly regard the monetary policy uncertainty, GDP report, and other macroeconomic data to finalize future investment strategy.

The government is responsible for formulating strategies to control the stock market, commodity market, FX rate, and fixed income securities market. The policy uncertainty concerning government regulation and congressional decision influence the behaviour of the investing community. The empirical outcome shows that EPU and VIX are expressively related, and the degree of association is on the higher side through the hard-monetary policy and fiscal clashes. The current work is limited to VIX and EPU indexes, and similar work further can be extended using the Equity Market Volatility (EMV) index as one of the proxies for market volatility uncertainty. The text-based equity market volatility (EMV) index, popularly known as policy news and stock market volatility indices. The relation between EMV index (e.g., specific to various markets) and VIX movements would yield some new insight about uncertainty studies.

\section{Acknowledgements}

We would like to thank anonymous reviewers, editor-in-chief and editorial office of Jr. of Business Economics and Management for the insightful suggestions and comments that has improved the work.

\section{References}

Alola, A. A., \& Uzune, G. (2020). The housing market and agricultural land dynamics: Appraising with economic policy uncertainty index. International Journal of Finance \& Economics, 25(2), 274-285. https://doi.org/10.1002/ijfe.1751 
Ashraf, B. N., \& Shen, Y. (2019). Economic policy uncertainty and banks' loan pricing. Journal of Financial Stability, 44, 100695. https://doi.org/10.1016/j.jfs.2019.100695

Badshah, I., Demirer, R., \& Suleman, M. T. (2019). he effect of economic policy uncertainty on stockcommodity correlations and its implications on optimal hedging. Energy Economics, 84, 104553. https://doi.org/10.1016/j.eneco.2019.104553

Baker, S. R., Bloom, N., \& Davis, S. J. (2016). Measuring economic policy uncertainty. The Quarterly Journal of Economics, 131(4), 1593-1636. https://doi.org/10.1093/qje/qjw024

Baker, S. R., Bloom, N., Davis, S. J., \& Kost, K. (2019, March 25). Policy news and stock market volatility. http://www.policyuncertainty.com/media/Policy\%20News\%20and\%20Stock\%20Market\%20 Volatility.pdf

Bali, T. G., Brown, S. J., \& Tang, Y. (2017). Is economic uncertainty priced in the cross-section of stock returns? Journal of Financial Economics, 126(3), 471-489. https://doi.org/10.1016/j.jineco.2017.09.005

Chen, E.-T. J., \& Clements, A. (2007). S\&P 500 implied volatility and monetary policy announcements. Finance Research Letters, 4(4), 227-232. https://doi.org/10.1016/j.frl.2007.07.002

Christensen, B., \& Prabhala, N. (1998). The relation between implied and realized volatility. Journal of Financial Economics, 50(2), 125-150. https://doi.org/10.1016/S0304-405X(98)00034-8

Christou, C., Cunado, J., Gupta, R., \& Hassapis, C. (2017). Economic policy uncertainty and stock market returns in PacificRim countries: Evidence based on a Bayesian panel VAR model. Journal of Multinational Financial Management, 40, 92-102. https://doi.org/10.1016/j.mulfin.2017.03.001

Degiannakis, S., \& Filis, G. (2019). Forecasting European economic policy uncertainty. Scottish Journal of Political Economy, 66(1), 94-114. https://doi.org/10.1111/sjpe.12174

Demir, E., Gozgor, G., Lau, C. K., \& Vigne, S. A. (2018). Does economic policy uncertainty predict the Bitcoin returns? An empirical investigation. Finance Research Letters, 26, 145-149. https://doi.org/10.1016/j.frl.2018.01.005

Duan, Y., Chen, W., Zeng, Q., \& Liu, Z. (2018). Leverage effect, economic policy uncertainty and realized volatility with regime switching. Physica A: Statistical Mechanics and its Applications, 493, 148-154. https://doi.org/10.1016/j.physa.2017.10.040

Engle, R. (2001). The use of ARCH/GARCH models in applied econometrics. Journal of Economic Perspectives, 15(4), 157-168. https://doi.org/10.1257/jep.15.4.157

Farka, M., \& Fleissig, A. R. (2012). The effect of FOMC statements on asset prices. International Review of Applied Economics, 26(3), 387-416. https://doi.org/10.1080/02692171.2011.587111

Federal Open Market Committee. (2009, December 16). Minutes of the December 2009 Meeting. http://www.federalreserve.gov/monetarypolicy/fomcminutes20091216.htm

Gábor, E., \& Georgarakos, D. (2018). Economic policy uncertainty and stock market participation (CFS Working Paper No. 590). https://doi.org/10.2139/ssrn.3139007

Ghirelli, C., Pérez, J. J., \& Urtasun, A. (2019). A new economic policy uncertainty index for Spain. Economics Letters, 182, 64-67. https://doi.org/10.1016/j.econlet.2019.05.021

Graham, M., Nikkinen, J., \& Sahlström, P. (2003). Relative importance of scheduled macroeconomic news for stock market investors. Journal of Economics and Finance, 27(2), 153-165. https://doi.org/10.1007/BF02827216

Hu, Z., Kutan, A. M., \& Sun, P.-W. (2018). Is US economic policy uncertainty priced in China's A-shares market? Evidence from market, industry, and individual stocks. International Review of Financial Analysis, 57, 207-220. https://doi.org/10.1016/j.irfa.2018.03.015

Huang, Y., \& Luk, P. (2020). Measuring economic policy uncertainty in China. China Economic Review, 59, 101367. https://doi.org/10.1016/j.chieco.2019.101367

Husted, L., Rogers, J., \& Sun, B. (2019). Monetary policy uncertainty. Journal of Monetary Economics (in Press). https://doi.org/10.1016/j.jmoneco.2019.07.009 
International Monetary Fund. (2012, October). World Economic Outlook: Coping with High Debt and Sluggish Growth. IMF Press.

Knapkova, M., Kiaba, M., \& Hudec, S. (2019). Impact of macroeconomic indicators on public debt of Slovak Republic. Journal of Business Economics and Management, 20(4), 734-753.

https://doi.org/10.3846/jbem.2019.10184

Nana, G.-A. N., Korn, R., \& Erlwein-Saye, C. (2013). GARCH-extended models: theoretical properties and applications. https://arxiv.org/abs/1307.6685

Nikkinen, J., \& Sahlström, P. (2004a). Scheduled domestic and US macroeconomic news and stock valuation in Europe. Journal of Multinational Financial Management, 14(3), 201-215. https://doi.org/10.1016/j.mulfin.2003.01.001

Nikkinen, J., \& Sahlström, P. (2004b). Impact of the federal open market committee's meetings and scheduled macroeconomic news on stock market uncertainty. International Review of Financial Analysis, 13(1), 1-12. https://doi.org/10.1016/j.irfa.2004.01.001

Nikkinen, J., Omran, M., Petri, S., \& Äijö, J. (2006). Global stock market reactions to scheduled U.S. macroeconomic news announcements. Global Finance Journal, 17(1), 92-104. https://doi.org/10.1016/j.gf.2006.06.003

Onan, M., Salih, A., \& Yasar, B. (2014). Impact of macroeconomic announcements on implied volatility slope of SPX options and VIX. Finance Research Letters, 11(4), 454-462. https://doi.org/10.1016/j.frl.2014.07.006

Pástor, L., \& Veronesi, P. (2012). Uncertainty about government policy and stock prices. The Journal of Finance, 67(4), 1219-1264. https://doi.org/10.1111/j.1540-6261.2012.01746.x

Pástor, L., \& Veronesi, P. (2013). Political uncertainty and risk premia. Journal of Financial Economics, 110(3), 520-545. https://doi.org/10.1016/j.jfineco.2013.08.007

Raza, S. A., Zaighum, I., \& Shah, N. (2018). Economic policy uncertainty, equity premium and dependence between their quantiles: Evidence from quantile-on-quantile approach. Physica A: Statistical Mechanics and its Applications, 492, 2079-2091. https://doi.org/10.1016/j.physa.2017.11.125

Reinhart, V., \& Simin, T. (1997). The market reaction to Federal Reserve policy action from 1989 to 1992. Journal of Economics and Business, 49(2), 149-168. https://doi.org/10.1016/S0148-6195(96)00077-X

Rigobon, R., \& Sack, B. (2004). The impact of monetary policy on asset prices. Journal of Monetary Economics, 51(8), 1553-1575. https://doi.org/10.1016/j.jmoneco.2004.02.004

Shaikh, I. (2017). The 2016 U.S. presidential election and the Stock, FX and VIX markets. The North American Journal of Economics and Finance, 42, 546-563. https://doi.org/10.1016/j.najef.2017.08.014

Shaikh, I. (2019a). On the relationship between economic policy uncertainty and the implied volatility index. Sustainability, 11(6), 1628. https://doi.org/10.3390/su11061628

Shaikh, I. (2019b). The relation between implied volatility index and crude oil prices. Engineering Economics, 30(5), 556-566. https://doi.org/10.5755/j01.ee.30.5.21611

Shaikh, I. (2020). Policy uncertainty and Bitcoin returns. Borsa Istanbul Review (in press). https://doi.org/10.1016/j.bir.2020.02.003

Wang, G.-J., Xie, C., Wen, D., \& Zhao, L. (2019). When Bitcoin meets economic policy uncertainty (EPU): Measuring risk spillover effect from EPU to Bitcoin. Finance Research Letters, 31, 489-497. https://doi.org/10.1016/j.frl.2018.12.028

Wang, S., \& Mayes, D. G. (2012). Monetary policy announcements and stock reactions: An international comparison. The North American Journal of Economics and Finance, 23(2), 145-164. https://doi.org/10.1016/j.najef.2012.02.002

Whaley, R. E. (2000). The investor fear gauge. The Journal of Portfolio Management, 26(3), 12-17. https://doi.org/10.3905/jpm.2000.319728

Witkowska, D., Kompa, K., \& Mente, G. (2019). The effect of government decisions on the efficiency of the investment funds market in Poland. Journal of Business Economics and Management, 20(3), 573-594. https://doi.org/10.3846/jbem.2019.9861 


\section{APPENDIX}

Table A.1. Description of various market volatility indices

\begin{tabular}{|c|c|c|c|c|}
\hline \multirow{2}{*}{$\begin{array}{l}\text { Sr. } \\
\text { No. }\end{array}$} & \multicolumn{4}{|c|}{ U.S. Market Volatility Indexes $\dagger$} \\
\hline & & Symbol & Underlying & Sample Period \\
\hline & \multicolumn{4}{|l|}{ Equity market volatility Index ${ }^{\infty}$} \\
\hline 1 & CBOE Volatility Index & VIX & S\&P500 & 01/2000-03/2018 \\
\hline 2 & CBOE NASDAQ Volatility Index & VXN & NASDAQ & 01/2001-03/2018 \\
\hline 3 & CBOE S\&P 100 Volatility Index & VXO & OEX & $01 / 2000-03 / 2018$ \\
\hline 4 & CBOE DJIA Volatility Index & VXD & DJIA & 01/2000-03/2018 \\
\hline \multirow[t]{2}{*}{5} & Volatility of VIX & VVIX & VIX & $01 / 2007-03 / 2018$ \\
\hline & Volatility Indexes on Commodity & & & \\
\hline 6 & NYMEX Crude Oil (WTI) Volatility Index & OIV & WTI & 09/2010-03/2018 \\
\hline 7 & CBOE Crude Oil ETF Volatility Index & OVX & USO & 05/2007-03/2018 \\
\hline 8 & CBOE Gold ETF Volatility Index & GVZ & SPDR-GLD & 01/2009-03/2018 \\
\hline 9 & CBOT Soybean Volatility Index & SIV & $\begin{array}{l}\text { US Soybeans } \\
\text { Futures }\end{array}$ & $06 / 2011-03 / 2018$ \\
\hline \multirow[t]{2}{*}{10} & CBOT Corn Volatility Index & CIV & $\begin{array}{l}\text { US Corn } \\
\text { Futures }\end{array}$ & $06 / 2011-03 / 2018$ \\
\hline & \multicolumn{4}{|l|}{ Volatility Indexes on Currency } \\
\hline 11 & CME FX Euro Volatility Index & EUVIX & FXE & 01/2007-03/2018 \\
\hline 12 & CME FX Yen Volatility Index & JYVIX & FXY & $01 / 2007-03 / 2018$ \\
\hline \multirow[t]{2}{*}{13} & CME FX British Pound Volatility Index & BPVIX & FXB & $01 / 2007-03 / 2018$ \\
\hline & Volatility Indexes on Interest Rates & & & \\
\hline 14 & $\begin{array}{l}\text { CBOT } 10 \text {-year U.S. Treasury Note } \\
\text { Volatility Index }\end{array}$ & TYVIX & T-Note futures & 01/2003-03/2018 \\
\hline
\end{tabular}

Table A.2. Description of scheduled macroeconomic announcements

\begin{tabular}{|c|l|l|l|}
\hline $\begin{array}{c}\text { Sr. } \\
\text { No. }\end{array}$ & \multicolumn{1}{|l|}{ Important Events (Economic and Political) $\dagger \dagger$} & \multicolumn{1}{|c|}{ Frequency } & \# Reports \\
\hline 1 & FOMC meeting & Quarterly & 147 \\
\hline 2 & GDP & Quarterly & 256 \\
\hline 3 & Other Macroeconomic indicators & Quarterly/Monthly & 2444 \\
\hline
\end{tabular}

Source: †Cboe Options Exchange (Cboe) http://www.cboe.com/products/vix-index-volatility/volatility-indexes $\dagger \dagger$ Federal Reserve Bank of St. Louis, https://alfred.stlouisfed.org/releases?pageID=1 